\title{
A Model for the Wind-Driven Current in the Wavy Oceanic Surface Layer: Apparent Friction Velocity Reduction and Roughness Length Enhancement
}

\author{
Miguel A. C. TeIXEIRA \\ Department of Meteorology, University of Reading, Reading, United Kingdom
}

(Manuscript received 24 April 2018, in final form 17 September 2018)

\begin{abstract}
A simple analytical model is developed for the current induced by the wind and modified by surface wind waves in the oceanic surface layer, based on a first-order turbulence closure and including the effect of a vortex force representing the Stokes drift of the waves. The shear stress is partitioned between a component due to shear in the current, which is reduced at low turbulent Langmuir number $\mathrm{La}_{t}$, and a wave-induced component, which decays over a depth proportional to the dominant wavelength $\lambda_{w}$. The model reproduces the apparent reduction of the friction velocity and enhancement of the roughness length estimated from current profiles, detected in a number of studies. These effects are predicted to intensify as $\mathrm{La}_{t}$ decreases and are entirely attributed to nonbreaking surface waves. The current profile becomes flatter for low $\mathrm{La}_{t}$ owing to a smaller fraction of the total shear stress being supported by the current shear. Comparisons with the comprehensive dataset provided by the laboratory experiments of Cheung and Street show encouraging agreement, with the current speed normalized by the friction velocity decreasing as $\mathrm{La}_{t}$ decreases and $\lambda_{w}$ increases if the model is adjusted to reflect the effects of a full wave spectrum on the intensity and depth of penetration of the wave-induced stress. A version of the model where the shear stress decreases to zero over a depth consistent with the measurements accurately predicts the surface current speed. These results contribute toward developing physically based momentum flux parameterizations for the wave-affected boundary layer in ocean circulation models.
\end{abstract}

\section{Introduction}

Flow coupling across the air-water interface in oceanic regions takes place within boundary layers where various properties adjust, over a relatively small fraction of the depth of the atmosphere and ocean, between their values in the interior of each fluid. The atmospheric and oceanic surface layers are the sublayers of these boundary layers located nearest to the air-water interface, occupying about $10 \%$ of their depth, which have a decisive importance in mediating the turbulent fluxes of momentum, heat, and gases between the atmosphere and the ocean (Csanady 2004), and where these fluxes are approximately constant. Hereafter, "surface layer" will always be used with this meaning, although the term is often adopted in an oceanographic context to denote the whole oceanic boundary layer.

Whereas the atmospheric surface layer over land has a no-slip bottom boundary condition applied at the

Corresponding author: Miguel A. C. Teixeira, m.a.teixeira@ reading.ac.uk ground, the atmospheric and oceanic surface layers in ocean regions are characterized by continuity of velocity and stress at the mobile air-water interface that separates them. This, on the one hand, leads to the generation of a wind-induced current in the oceanic surface layer, and on the other hand allows the generation of surface waves at the air-water interface. Both of these aspects considerably complicate the physics of these surface layers, especially the oceanic one, as is widely recognized (Thorpe 2005) and will be further discussed here.

Nevertheless, the oceanic surface layer is still largely understood and modeled based on the transposition to the ocean of theories developed for the atmospheric surface layer over land, where the effects of surface waves are not represented (Kraus and Businger 1994). Deficiencies in this approach become apparent when one realizes that key parameters in surface layer theory, such as the friction velocity $u *$ and roughness length $z_{0}$, are deemed to take values in the ocean that seem to be inconsistent with the values of the shear stress and the geometric properties of the air-water interface, respectively. 
Standard surface layer theory is based on MoninObukhov scaling, which in the limit of neutral stratification reduces to a theory for the logarithmic mean wind profile. In the ocean, or in underwater flows measured in the laboratory, such a theory has been applied, with varying degrees of success, to model the mean current induced by the wind. However, it has often been detected that the value of $u *$ inferred from the current profile is noticeably smaller than the one that would be expected from the total shear stress (McWilliams et al. 1997; Kudryavtsev et al. 2008; Teixeira and Belcher 2010), a phenomenon sometimes alternatively interpreted as an increase of the von Kármán parameter (Howe et al. 1982; Cheung and Street 1988; Craig and Banner 1994; Siddiqui and Loewen 2007). On the other hand, the value of $z_{0}$ obtained by extrapolating the logarithmic current profile up to the surface is often much larger than would be expected based on the size of the surface corrugations deforming the air-water interface, and exceeds by several orders of magnitude the air-side value of $z_{0}$ (Csanady 1984; Burchard 2001; Soloviev and Lukas 2003; Sullivan et al. 2004; Kudryavtsev et al. 2008).

There is some awareness that the first aspect is due to the fact that a fraction of the surface stress is carried by surface waves, and therefore does not support as much shear as if the waves were absent. On the other hand, the increased values of $z_{0}$ have been attributed to the effect of surface waves as roughness elements seen from below, or to wave breaking, but the exact mechanism by which this enhancement arises remains rather mysterious. The huge disparity between the estimated values of $z_{0}$ as seen from the air side or from the water side of the air-water interface is especially puzzling, since the amplitude of the corrugations is the same. Even if the flow on both sides of the air-water interface could be assumed to be aerodynamically smooth, the differences in the value of $u *$ between air and water would not be enough to explain the magnitude of this disparity.

Craig and Banner (1994) and Craig (1996) developed a model of the oceanic surface layer that produces profiles of the mean current and of the associated dissipation rate of turbulent kinetic energy (TKE), which showed some success in predicting both quantities, and was subsequently adapted and used by a number of researchers (e.g., Drennan et al. 1996; Terray et al. 1999; Gemmrich and Farmer 1999; Burchard 2001; Rascle et al. 2006; Feddersen et al. 2007; Rascle and Ardhuin 2009; Gerbi et al. 2009; Kukulka and Harcourt 2017). That model is based on an approximate balance between the turbulent fluxes of TKE and dissipation and produces a substantial surface dissipation enhancement, which is consistent with the observations of Gargett (1989), Agrawal et al. (1992), Terray et al. (1996), and Drennan et al. (1996). However, it requires adjusting $z_{0}$ for each dataset, yielding values of this quantity on the order of the height or wavelength of the surface waves, which is much larger than estimated for an aerodynamically smooth boundary, or from the Charnock relation. Both Craig and Banner (1994) themselves and, more recently, Grant and Belcher (2009) recognized that this need to adjust $z_{0}$ in order to fit measurements is a weakness of the model.

More recently, Kudryavtsev et al. (2008) developed a rather elaborate model based on the momentum and TKE budgets and assuming a balance between turbulence production by wave breaking and dissipation. This model avoids the strong dependence on $z_{0}$ displayed by the model of Craig and Banner (1994), but contains many ad hoc assumptions and approximations (e.g., the parameterization of the TKE production by wave breaking, or the mixing length definition), and nevertheless is so complicated that the corresponding equations can only be solved numerically. Although it predicts satisfactorily the qualitative behavior of the mean current profiles measured in the laboratory experiments of Cheung and Street (1988) and the aforementioned surface dissipation enhancement, it produces dissipation profiles that look somewhat artificial and seem to underestimate most datasets at small depths (see their Fig. 7). Although this model succeeds in predicting the enhanced values of the apparent $z_{0}$ in the experiments of Cheung and Street (1988), it does not explain the reduced values of $u_{*}$ that can also be inferred from the slope of the mean flow profiles.

In this study a very simple model is developed, based on the partition of the shear stress in the surface layer between shear-related and wave-related parts, that reconciles all these results, explaining in particular the discrepancies between expected and observed values of $u *$ and $z_{0}$ in the oceanic surface layer, purely due to the effect of nonbreaking waves (unlike Kudryavtsev et al. 2008). The model draws heavily on that developed by Teixeira (2012), which is inspired by rapid distortion theory (RDT) calculations, and is essentially analytical, being much simpler than the one proposed by Kudryavtsev et al. (2008), but is able to produce more accurate results. It has the advantage of being formulated as a variant of Monin-Obukhov scaling, where instead of the Obukhov stability parameter, the key dimensionless parameters account for the effects of surface waves. These parameters are the well-known turbulent Langmuir number $\mathrm{La}_{t}$ and (as in Monin-Obukhov theory) a dimensionless depth, here normalized by the wavenumber of the dominant surface waves. An extended version of this model was shown by Teixeira (2012) to give good predictions of the dissipation rate by comparison with field data from various sources (Terray et al. 1996; Drennan et al. 1996; Burchard 2001; Feddersen et al. 2007; Jones and 
Monismith 2008; Gerbi et al. 2009). The model is tested here by comparison with the data of Cheung and Street (1988), showing good agreement, despite the fact that [unlike the model of Kudryavtsev et al. (2008)] it uses a monochromatic wave approximation and neglects the viscous boundary layer.

This paper is organized as follows: section 2 presents the proposed model, including its version for a vertically uniform shear stress and its extension for a shear stress that decreases linearly with depth. Section 3 contains the results, starting with tests to the model as a function of its input parameters, and proceeding with its comparison with laboratory data. Finally, in section 4, the main conclusions of this study are summarized.

\section{Theoretical model}

It will be assumed that the rotation of Earth and stratification of the water in the oceanic surface layer can be neglected. The first assumption is generally acceptable in the surface layer, where the flow is by definition dominated by turbulent fluxes (and throughout the whole oceanic boundary layer in equatorial regions, where the Coriolis parameter is zero). The second assumption is acceptable if some other dynamical process (in the present case, the effect of surface waves) is stronger than that of buoyancy. The effect of breaking surface waves will also be neglected. This is a working hypothesis, which is not as justifiable as the previous two, but was shown to be a plausible approximation given the level of agreement achieved between the model of Teixeira (2012) and dissipation data (for further details concerning its motivation, see that paper).

The water-side friction velocity $u *$ and roughness length $z_{0}$ will be specified according to their most fundamental definitions: as the square root of the surface value of the kinematic shear stress and as the depth at which the current velocity relative to its surface value is zero (without assuming a displacement height), respectively, rather than based on the slope and intercept of the current profiles (which would be misleading in the present context).

The point of departure for the model is that turbulence in the surface layer is dominated by the transfer of kinetic energy from the mean wind-driven current and the Stokes drift of surface waves to the turbulence, via the shear production and Stokes drift production terms in the TKE budget (see, e.g., McWilliams et al. 1997), which are assumed to be balanced locally by the dissipation rate, as in Teixeira (2011b, 2012). This balance, although of questionable accuracy, has been motivated in Teixeira (2012) by the TKE budgets presented in the large-eddy simulation (LES) studies of Polton and Belcher (2007), Grant and Belcher (2009), and Kukulka et al. (2010) (which did not account for the effects of wave breaking). More recent supporting evidence for this balance is provided by Van Roekel et al. (2012) and Kukulka and Harcourt (2017).

RDT studies (e.g., Lee et al. 1990; Teixeira and Belcher 2002, 2010; Teixeira 2011a) have indicated that the characteristics of the turbulence (i.e., its anisotropy and rate of energy transfer from the mean flow) are determined by its distortion by the mean current shear $d U / d z$ [where $U(z)$ is the mean current speed], which promotes horizontal "streaky structures," and by the Stokes drift gradient $d U_{S} / d z$ [where $U_{S}(z)$ is the Stokes drift velocity], which promotes instead streamwise vortices with strong vertical velocity fluctuations. The influence of surface waves can be measured by the relative importance of these two strain rates, since the corresponding production terms in the TKE budget may be written (for a wind stress aligned in the $x$ direction)

$$
-\overline{u^{\prime} w^{\prime}} \frac{d U}{d z}, \quad-\overline{u^{\prime} w^{\prime}} \frac{d U_{S}}{d z},
$$

where $\tau / \rho=-\overline{u^{\prime} w^{\prime}}$ is the kinematic shear stress (with $u^{\prime}$ and $w^{\prime}$ being the horizontal and vertical turbulent velocity fluctuations, respectively), and $\rho$ is the density. It will be assumed hereafter that $d U / d z$ and $d U_{S} / d z$ have the same sign $(>0)$, which is the typical situation for wind-driven waves.

\section{a. Scaling of the oceanic surface layer}

The vertical gradient of the Stokes drift of a deepwater monochromatic surface wave of amplitude $a_{w}$, wavenumber $k_{w}$, and angular frequency $\sigma_{w}$ at a depth $z$ is given by (Phillips 1977)

$$
\frac{d U_{S}}{d z}=2\left(a_{w} k_{w}\right)^{2} \sigma_{w} e^{-2 k_{w}|z|},
$$

and, to a first approximation, in the surface layer the mean current shear satisfies

$$
\frac{d U}{d z}=\frac{u_{*}}{\kappa|z|},
$$

where $\kappa$ is the von Kármán constant. To evaluate the relative importance of the Stokes drift strain rate and mean shear rate of the current, the ratio of (2) and (3) may be taken at a representative depth where the flow is affected by surface waves, say $|z|=1 /\left(2 k_{w}\right)$, yielding

$$
\begin{aligned}
R & =\frac{d U_{S} / d z}{d U / d z}\left[|z|=1 /\left(2 k_{w}\right)\right]=\kappa e^{-1}\left(a_{w} k_{w}\right)^{2} \frac{c_{w}}{u_{*}} \\
& =\kappa e^{-1} \frac{U_{S}(z=0)}{u_{*}}=\kappa e^{-1} \mathrm{La}_{t}^{-2},
\end{aligned}
$$

where $U_{S}(z=0)=\left(a_{w} k_{w}\right)^{2} c_{w}$ is the Stokes drift velocity at the surface, $c_{w}=\sigma_{w} / k_{w}$ is the phase speed of the 
waves, and $\mathrm{La}_{t}=\left[u_{*} / U_{S}(z=0)\right]^{1 / 2}$ is the turbulent Langmuir number. Incidentally, $|z|=1 /\left(2 k_{w}\right)$ is also the depth at which $R$ attains its maximum (cf. Teixeira and Belcher 2010; Teixeira 2011a).

Consider first the magnitude of $R$ in the atmosphere. Although one does not often think about Stokes drift in the atmosphere, its magnitude is similar to that in the ocean, since the wave orbital motions (usually immersed in a tangle of turbulent eddies) are likewise of similar magnitude. Parameter $d U_{S} / d z$ is estimated here as if $d U / d z$ did not affect the wave motion, which is certainly not strictly true, but provides a leading-order approximation. For waves of slope $a_{w} k_{w} \approx 0.1$ and wavelengths in the range $\lambda_{w} \approx 1-100 \mathrm{~m}$, taking into account that $k_{w}=2 \pi / \lambda_{w}$, then the wavenumber is in the range $k_{w} \approx 0.06-6.3 \mathrm{~m}^{-1}$, and using the linear dispersion relation of deep-water gravity waves, $c_{w}=\sqrt{g / k_{w}}$, one obtains $c_{w} \approx 1.25-12.5 \mathrm{~m} \mathrm{~s}^{-1}$, with the limits swapped relative to those of $k_{w}$. Taking a typical value of the friction velocity in the atmosphere, $u * \approx$ $0.3 \mathrm{~m} \mathrm{~s}^{-1}$, (4) yields $R \approx 6 \times 10^{-3}-6 \times 10^{-2}$ (where $\kappa=0.4$ was assumed), which is very small. This means, perhaps unsurprisingly, that the effect of the Stokes drift in the atmosphere is fairly insignificant, and the surface layer should be dominated by mean wind shear.

For the oceanic surface layer, although the same estimates for the wave characteristics may be used, it must be noted that, to a first approximation, the shear stress $\tau$ is continuous across the air-water interface in steady flow, and since by definition $\overline{u^{\prime} w^{\prime}}(z=0)=-u_{*}^{2}$, then $\rho u_{*}^{2}$ must be continuous at that interface. Given that the density ratio between water and air is $\approx 833$, the friction velocity in the water will be smaller by a factor of $\sqrt{833} \approx 29$. This gives a typical friction velocity of $u_{*} \approx 0.01 \mathrm{~m} \mathrm{~s}^{-1}$, yielding $R \approx 0.17-1.7$, which is of $O(1)$. In reality, the value of $u *$ used in (3) should be even smaller, since part of the shear stress is supported by the wave as well as by the mean shear (as will be seen later), so that it is common to have $R$ substantially higher than 1 . In addition, it is quite possible that $a_{w} k_{w}>0.1$, which also increases $R$. This means that in the ocean it is unacceptable to ignore the effect of the Stokes drift of surface waves, and this difference is what gives oceanic turbulence its distinctive character, as shown by McWilliams et al. (1997) using LES and Teixeira and Belcher $(2002,2010)$ and Teixeira (2011a) using RDT.

\section{b. Shear stress partition}

The Craik-Leibovich equations including the effect of the Stokes drift of surface waves may be manipulated, in the same way as done for obtaining a TKE budget including the production terms in (1), to obtain an equation for evolution of the shear stress (Teixeira 2011a):

$$
\frac{d}{d t} \overline{u^{\prime} w^{\prime}}=-\overline{w^{\prime 2}} \frac{d U}{d z}-\overline{u^{\prime 2}} \frac{d U_{S}}{d z}+\text { other terms. }
$$

This equation shows that the shear stress receives contributions proportional to the mean shear and to the Stokes drift strain rate. This prompted Teixeira (2011a) to decompose $\overline{u^{\prime} w^{\prime}}$ into shear-induced and wave-induced components, proportional to the corresponding production terms explicitly presented in (5). Hence, the shear-induced component of $\overline{u^{\prime} w^{\prime}}$ can be parameterized as

$$
\begin{aligned}
\left(\overline{u^{\prime} w^{\prime}}\right)_{s} & =\overline{u^{\prime} w^{\prime}} \frac{\overline{w^{\prime 2}} d U / d z}{\overline{w^{\prime 2}} d U / d z+\overline{u^{\prime 2}} d U_{S} / d z}=\frac{\overline{\overline{u^{\prime} w^{\prime}}}}{1+\frac{\overline{u^{\prime 2}}}{\overline{w^{\prime 2}}} \frac{d U_{S} / d z}{d U / d z}} \\
& =\frac{\overline{u^{\prime} w^{\prime}}}{1+\frac{\overline{u^{\prime 2}}}{\overline{w^{\prime 2}}} 2 \kappa\left(a_{w} k_{w}\right)^{2} \frac{c_{w}}{u_{*}} k_{w}|z| e^{-2 k_{w}|z|}}
\end{aligned}
$$

where (2) and (3) have been used. Although the logarithmic current profile is modified by wave effects (as shown in the following section) and therefore (3) is not strictly accurate, it provides a correct scaling which makes the proposed shear stress partition both be simple enough and benefit from good properties. The inaccuracy of this approximation is likely to partially cancel with those of other adopted assumptions, as noted below. Using the definitions of $u *, U_{S}(z=0)$, and $\mathrm{La}_{t}$, (6) may be alternatively expressed as

$$
\begin{aligned}
\left(\overline{u^{\prime} w^{\prime}}\right)_{s} & =-\frac{u_{*}^{2}}{1+2 \kappa \overline{\overline{u^{\prime 2}}} \frac{U_{S}(z=0)}{w^{\prime 2}} k_{w}|z| e^{-2 k_{w}|z|}} \\
& =-\frac{u_{*}^{2}}{1+2 \kappa \frac{\overline{u^{\prime 2}}}{\overline{w^{\prime 2}}} \operatorname{La}_{t}^{-2} k_{w}|z| e^{-2 k_{w}|z|}},
\end{aligned}
$$

where it has been noted that in the surface layer the shear stress $\overline{u^{\prime} w^{\prime}}$ is constant and equal to $-u_{*}^{2}$. If, following Teixeira (2012), it is assumed that the quantity $\gamma=2 \kappa\left(k_{w}|z|\right)\left(\overline{u^{12}} / w^{\prime 2}\right)$ is approximately constant (which has some plausibility given that $\overline{w^{2}}$ must approach zero as $z \rightarrow 0$, particularly in a curvilinear wave-following coordinate system; cf. Teixeira and Belcher 2002), then the shear-induced shear stress takes the form

$$
\left(\overline{u^{\prime} w^{\prime}}\right)_{s}=-\frac{u_{*}^{2}}{1+\gamma \mathrm{La}_{t}^{-2} e^{-2 k_{w}|z|}},
$$

where $\gamma$ is an adjustable (positive) coefficient. The calibration of this coefficient may be exploited to account for extraneous effects, such as the possibility that the 
waves are nonmonochromatic, and the fact that the current profile is not perfectly logarithmic. Assuming that $\gamma$ is constant with depth is likely to be less accurate outside the surface layer, because the above assumptions about the behavior of $\overline{w^{2}}$ and $d U / d z$ as $z \rightarrow 0$ do not hold anymore, but the model is not applicable there anyway.

Note that (8) has the properties of approaching the usual definition of the total shear stress as either $|z| \rightarrow \infty$ or $\mathrm{La}_{t} \rightarrow \infty$, both of which make sense physically. The usual wall-layer scaling for the dissipation rate, consistent with (3) and with a logarithmic current profile, was shown to hold by the observations of various authors at sufficiently large depths (Gargett 1989; Agrawal et al. 1992; Terray et al. 1996) and is obviously recovered when the influence of surface waves becomes vanishingly small (which corresponds to $\mathrm{La}_{t} \rightarrow \infty$; McLeish and Putland 1975; Kondo 1976). The remaining part of the shear stress, $\overline{u^{\prime} w^{\prime}}-\left(\overline{u^{\prime} w^{\prime}}\right)_{s}$, is evidently wave related and approaches zero when either $|z| \rightarrow \infty$ or $\mathrm{La}_{t} \rightarrow \infty$. Its depth of penetration is clearly, from (8), of $O\left[1 /\left(2 k_{w}\right)\right]$, although it should be borne in mind that this particular dependence results directly from the monochromatic wave approximation. Other approaches to treat the dependence of $\left(d U_{S} / d z\right) /(d U / d z)$ (as well as that of $\overline{u^{\prime 2}} / \frac{1 w^{\prime 2}}{)}$ with depth could result in different functional forms for $\left(\overline{u^{\prime} w^{\prime}}\right)_{s}$, with $\gamma$ possibly not being treated as a constant.

An interesting property of (8) is that, when evaluated at the surface, it allows the definition of a modified friction velocity affected by shear $u_{*_{s}}$ as

$$
u_{*_{s}}=-\frac{\left(\overline{u^{\prime} w^{\prime}}\right)_{s}}{u_{*}}=\frac{u_{*}}{1+\gamma \mathrm{La}_{t}^{-2}} .
$$

Clearly, $u *_{s}$ is always smaller than $u *$ and can even become much smaller when $\mathrm{La}_{t}$ is low. This is in agreement with LES results by, for example, McWilliams et al. (1997), Li et al. (2005), and Grant and Belcher (2009) showing that shear in the current profile decreases markedly for a constant wind stress $\tau$ as $\mathrm{La}_{t}$ decreases (see section 3). One advantage of (9) is that it allows the definition of friction velocities due to shear and due to the wave that are additive, yielding the sum $u *$. The present approach partially resembles the modification of the surface shear stress to account for wave effects in the study of the Ekman-Stokes boundary layer by Polton et al. (2005), where, however, Earth's rotation effect was taken into account.

\section{c. A model for the current profile}

To obtain a model for the current profile that is consistent with the existing surface layer theory, a firstorder turbulence closure is applied to the shear-related part of the shear stress, namely,

$$
\left(\overline{u^{\prime} w^{\prime}}\right)_{s}=-K_{m} \frac{d U}{d z}
$$

where $K_{m}=\kappa u *|z|$, as usually defined. Here $u *$ is taken as the relevant velocity scale for momentum transport, since the vertical velocity fluctuations, which effect this transport, scale on $u_{*}$ rather than on $u_{*}$. Then the shear of the mean current can be expressed as

$$
\frac{d U}{d z}=-\frac{\left(\overline{u^{\prime} w^{\prime}}\right)_{s}}{\kappa u_{*}|z|}=\frac{u_{*}}{\kappa|z|} \phi_{L}\left(\mathrm{La}_{t}, k_{w}|z|\right),
$$

where (8) has been used in the second equality, and

$$
\phi_{L}\left(\mathrm{La}_{t}, k_{w}|z|\right)=\frac{1}{1+\gamma \mathrm{La}_{t}^{-2} e^{-\varepsilon k_{w}|z|}},
$$

where $\varepsilon=2$ from (8), but will hereafter be kept as an adjustable parameter for maximum generality. As for $\gamma$, the adjustment of $\varepsilon$ may be exploited to account for various extraneous effects, such as the presence of nonmonochromatic waves. The connection with this latter aspect is even closer, since $\varepsilon$ controls the vertical penetration of wave effects, which may depend not only on the dominant wavelength, but also on the wave energy distribution by scale.

Note that $\phi_{L}$ plays in (11) a role analogous to that played by stability functions in Monin-Obukhov theory of the nonneutral surface layer. The difference resides in the fact that $\phi_{L}$ depends on wave quantities [according to (12)] instead of on stratification. This formulation is amenable to improvement, since the form of (12) only needs to be modified to account for missing effects or a more accurate representation of the effects already considered. The form taken by (11) implies that both at large depths (where usual surface layer scaling is recovered) and near the surface $z \approx 0$ the current profile is approximately logarithmic, but with different friction velocities $u *$ and $u *_{s}$, respectively, as expressed by (9). The dependence of (12) on $z$ is, arguably, the simplest possible that benefits from these properties. The partition of the shear stress into shear-induced and wave-induced components, conjugated with the use of a first-order turbulence closure in (10), parallels the approach, used in a numerical modeling context, of Harcourt (2013). However, the partition itself was originally suggested by Teixeira (2011a) based on the shear stress budget in (5) and used in the present form by Teixeira (2012).

From (10)-(12) it is possible to define an "effective" eddy viscosity $K_{m}^{*}$ that takes into account wave effects:

$$
K_{m}^{*}=-\frac{\overline{u^{\prime} w^{\prime}}}{d U / d z}=\kappa u_{*}|z|\left(1+\gamma \mathrm{La}_{t}^{-2} e^{-\varepsilon k_{w}|z|}\right) .
$$


Its form clearly shows the apparent mixing enhancement resulting from the reduction of $d U / d z$ by those effects.

To complete the model, it remains to integrate (11) between $z=z_{0}$ (where $U=U_{0}, U_{0}$ being the Eulerian current at the surface) and a generic $z$. This yields

$$
U_{0}-U(z)=\frac{u_{*}}{\kappa} \int_{z_{0}}^{|z|} \frac{1}{z^{\prime}} \frac{1}{1+\gamma \mathrm{La}_{t}^{-2} e^{-\varepsilon k_{w} z^{\prime}}} d z^{\prime} .
$$

If velocities are normalized by $u_{*}$ and $|z|$ by $k_{w},(14)$ may be rewritten

$$
\frac{U_{0}-U(z)}{u_{*}}=\frac{1}{\kappa} \int_{k_{w} z_{0}}^{k_{w} z \mid} \frac{1}{z^{\prime}} \frac{1}{1+\gamma \mathrm{La}_{t}^{-2} e^{-\varepsilon z^{\prime}}} d z^{\prime} .
$$

Often, current profiles in the surface layer are specified using so-called wall coordinates, defined as $U^{+}=$ $\left[U_{0}-U(z)\right] / u_{*}$ and $z^{+}=|z| u_{*} / \nu$, where $\nu$ is the kinematic viscosity of water. Using these definitions, (15) can be expressed as

$$
U^{+}=\frac{1}{\kappa} \int_{\frac{k_{w} w_{*}}{u_{*}} \frac{z_{u^{\prime}} u_{*}}{\nu} z^{+}}^{\frac{k_{w}{ }^{+}}{z^{\prime}}} \frac{1}{1+\gamma \mathrm{La}_{t}^{-2} e^{-\varepsilon z^{\prime}}} d z^{\prime} .
$$

The advantage of expressing the lower limit of integration in this form is that for aerodynamically smooth flow, $z_{0} u_{*} / \nu=0.11$ (Cheung and Street 1988; Kraus and Businger 1994), a result that will be used below. The integral in (15) or (16) cannot in general be evaluated analytically. For numerical evaluation purposes only, it is useful to introduce the further change of variable $z^{\prime}=\exp \zeta$, which transforms (16) into

$$
U^{+}=\frac{1}{\kappa} \int_{\log \left(\frac{k_{w_{*}}{ }^{\nu}}{u_{*} z^{u_{*}}}\right)}^{\log \left(\frac{k_{w^{v}}{ }^{+}}{u^{+}}\right)} \frac{1}{1+\gamma \mathrm{La}_{t}^{-2} e^{-\varepsilon \exp \zeta}} d \zeta
$$

This eliminates the singularity at $z^{\prime}=0$, which is especially bothersome for small values of $z_{0}$.

In the limit $\mathrm{La}_{t} \rightarrow \infty$, (17) [or (16)] can, of course, be integrated analytically, reducing to

$$
U^{+}=\frac{1}{\kappa} \log \left(\frac{z^{+} \nu}{z_{0} u_{*}}\right)=\frac{1}{\kappa} \log \left(\frac{|z|}{z_{0}}\right) .
$$

For aerodynamically smooth flow, (18) further reduces to

$$
U^{+}=\frac{1}{\kappa} \log \left(\frac{z^{+}}{0.11}\right)=\frac{1}{\kappa} \log \left(z^{+}\right)+5.5,
$$

as noted by Cheung and Street (1988), where it was assumed that $\kappa=0.4$.
When plotted with a logarithmic scale for depth, (17) consists of two straight line segments separated by a transition depth interval centered around $|z| \approx 1 /\left(\varepsilon k_{w}\right)$. The slope of the current profile in its upper, waveaffected part, is consistent with the reduced friction velocity $u *_{s}$, given by (9), that is,

$$
\frac{d U}{d z}(z \rightarrow 0)=\frac{u_{*_{s}}}{\kappa|z|}
$$

[as results from (9), (11), and (12)], and $u *$ is of course consistent with the slope of the profile segment occurring at larger depths (see discussion below). The roughness length $z_{0}$ is the height at which $U^{+}=0$, irrespective of whether the current profile is affected by waves or not. In the latter case, an apparent roughness length can be defined, which corresponds to the intersect of the prolongation of the segment of the current profile at large depths with the axis where $U^{+}=0$. It can be anticipated that this apparent roughness length $z_{0 w}$ is much larger than the true $z_{0}$ when the effect of waves is important, because of the break point (or more precisely, transition region) existing in the current profile at $|z| \approx 1 /\left(\varepsilon k_{w}\right)$. Variable $z_{0 w}$ can be obtained by integrating (11) between $z_{0}$ and $\infty$ and then (3) back to $z_{0 w}$. This yields

$$
\begin{aligned}
\log \left(k_{w} z_{0 w}\right)= & \log \left(k_{w} z_{0}\right) \\
& +\gamma \mathrm{La}_{t}^{-2} \int_{\log \left(k_{w} z_{0}\right)}^{\infty} \frac{e^{-\varepsilon \exp \zeta}}{1+\gamma \mathrm{La}_{t}^{-2} e^{-\varepsilon \exp \zeta}} d \zeta .
\end{aligned}
$$

Equations (9), (17), and (21) form the basis of the calculations presented in this paper.

It is worth noting that the formulation of the shear stress on which these equations are based, (10), is strictly local, neglecting any transport effects, whereby $d U / d z$ might become negative with $\overline{u^{\prime} w^{\prime}}$ remaining also negative [corresponding to a negative eddy viscosity in (10)]. This behavior, which is produced in a number of LES results (McWilliams et al. 1997; Li et al. 2005; TejadaMartinez et al. 2013), was recently parameterized by Sinha et al. (2015) by adopting a nonlocal component of the shear stress, akin to those used in momentum flux parameterizations for convection. Since the data used in the present study (from Cheung and Street 1988) do not show such negative current shear [another example is the top surface layer in Fig. 5 of Longo et al. (2012)], that approach is not used here, although it may be viewed as one of the possible improvements to the present scheme.

\section{MODEL FOR A LINEARLY DECREASING SHEAR STRESS}

For the purpose of comparing the model developed above with the laboratory measurements of Cheung and 
Street (1988) (to be done below), it is convenient to assume that the shear stress is not constant with depth, but rather varies linearly from its maximum at the airwater interface to zero at a certain depth. This parallels the approach used by Cheung and Street (1988) to estimate the shear stress from their data, and corresponds mathematically to

$$
\overline{u^{\prime} w^{\prime}}=-u_{*}^{2}\left(1-\frac{|z|}{\delta}\right), \quad \text { if } \quad|z| \leq \delta,
$$

where $\delta$ is the depth where $\overline{u^{\prime} w^{\prime}}$ becomes zero, and it is implied that for $|z|>\delta, \overline{u^{\prime} w^{\prime}}=0$. In this case, the function $\phi_{L}$ must be redefined (for $|z| \leq \delta$ ) as

$$
\phi_{L}\left(\mathrm{La}_{t}, k_{w}|z|, \frac{|z|}{\delta}\right)=\frac{1-\frac{|z|}{\delta}}{1+\gamma \mathrm{La}_{t}^{-2} e^{-\varepsilon k_{w}|z|}},
$$

and (11) may then be integrated to give

$U^{+}=\frac{1}{\kappa} \int_{\frac{k_{w} w_{*}}{u_{*}}}^{\frac{k_{w w^{v}} z^{+}}{u_{*}} z^{+}}\left(\frac{1}{z^{\prime}}-\frac{1}{k_{w} \delta}\right) \frac{1}{1+\gamma \mathrm{La}_{t}^{-2} e^{-\varepsilon z^{\prime}}} d z^{\prime}$

(again valid only for $|z| \leq \delta$ ), replacing (16). For $|z|>\delta$, $U^{+}=U^{+}\left(z^{+}=\delta u * / \nu\right)$, which is constant. In the limit $\mathrm{La}_{t} \rightarrow \infty,(24)$ reduces to

$$
U^{+}=\frac{1}{\kappa}\left[\log \left(z^{+}\right)-\frac{z^{+}}{\frac{\delta u_{*}}{\nu}}-\log \left(\frac{z_{0} u_{*}}{\nu}\right)+\frac{z_{0}}{\delta}\right],
$$

which has a log-linear variation and must replace (19).

Note that, according to (10) and (22), for $|z|>\delta$, $d U / d z=0$ under the present assumptions, that is, no mean shear exists and the current speed does not vary. This gives the version of the model just described the capability of predicting the surface value of the windinduced current (unlike the version described in the previous subsection, where $U$ varies indefinitely). Defining arbitrarily $U(|z|=\delta)=0$, which makes sense since this is the value of the current at the depth where the effect of the surface wind stress is no longer felt, then from the definition of $U^{+}$it follows that $U_{0} / u_{*}=$ $U^{+}(|z|=\delta)=U^{+}\left(z^{+}=\delta u_{*} / \nu\right)$, which can be obtained from (24).

As a caution, it should be emphasized that the assumption of a nonconstant shear stress, expressed by (22), may not be strictly consistent with statistically steady and horizontally homogeneous flow (implicit in surface layer theory), requiring either a time evolution of the mean current or a mean horizontal pressure gradient, but hopefully this assumption is still acceptable for the present purposes. A model with a linearly decreasing shear stress, such as the one just presented, might be thought of as a very simple representation of the whole oceanic boundary layer (of depth $\delta$ ) instead of just the surface layer. However, its applicability to real cases is limited by neglect of the effect of Earth's rotation, the choices made to approximate (7) as (8), and the Monin-Obukhov approach inherent to (11) and (12). These are confined to the surface layer, and would require modification in order to extend the model.

\section{Results}

It is instructive first of all to explore the model behavior for a few representative cases, because this illustrates in the "cleanest" possible way the range of behavior of the model and its impact on the perceived values of the water-side values of $u *$ and $z_{0}$. More detailed comparisons with laboratory experiments follow. In all of these cases, $\gamma$ and $\varepsilon$ will be treated as adjustable parameters.

\section{a. Generic behavior of the model}

Figure 1 shows profiles of $U^{+}$as a function of $k_{w}|z|$ from (15) for $k_{w} z_{0}=0.001$ and different values of the turbulent Langmuir number $\mathrm{La}_{t}=0.5,1,2$, assuming that $\gamma=1$ and $\varepsilon=1$, for simplicity. Note that these values of $\gamma$ and $\varepsilon$ are of the same order of magnitude as those adopted by Teixeira (2012). The results are not qualitatively very sensitive to $k_{w} z_{0}$ in the representation adopted in Fig. 1, and variation of this parameter merely leads to a rescaling of the horizontal axis, with a narrower transition region between the two logarithmic portions of the curves occurring for values of $k_{w} z_{0} \ll 1$.

The value $\mathrm{La}_{t}=2$ intends to represent shear-dominated turbulence, $\mathrm{La}_{t}=0.5$ refers to Langmuir (i.e., wave dominated) turbulence, and $\mathrm{La}_{t}=1$ to turbulence with a transitional character. As can be seen in Fig. 1, the current profiles (denoted by the solid curves) have a lower portion with invariant slope for larger depths. This slope, when expressed in terms of $U^{+} / \log |z|$, is $1 / \kappa$, because of the way $U^{+}$ is normalized. At smaller depths the current profile has a lower slope (prolonged to larger $|z|$ as the dashed asymptotes), which is proportional to the values of the ratio $u_{*} / u_{*}$ in each case. From (9) (for $\gamma=1$ ), these values are $u_{*} / u_{*}=0.8$ for $\mathrm{La}_{t}=2, u_{*} / u_{*}=0.5$ for $\mathrm{La}_{t}=1$, and $u_{*} / u_{*}=0.2$ for $\mathrm{La}_{t}=0.5$. On the other hand, if the lower portion of the current profile is prolonged toward the surface (dotted line asymptotes), one obtains an "effective" value of the roughness length, expressed by (21), which would be obtained by ignoring the upper portion of the current profile. For $\mathrm{La}_{t}=2, k_{w} z_{0 w}=0.004$; for $\mathrm{La}_{t}=1$, $k_{w} z_{0 w}=0.030 ;$ and for $\mathrm{La}_{t}=0.5, k_{w} z_{0 w}=0.341$, which 


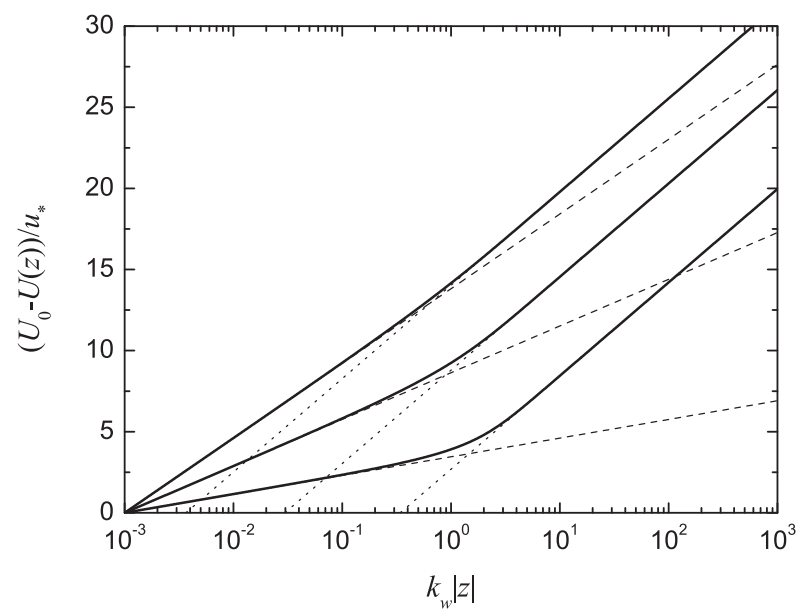

FIG. 1. Normalized current speed as a function of normalized depth for different values of $\mathrm{La}_{t}$, calculated from (15) for $\gamma=1, \varepsilon=1$, and $k_{w} z_{0}=0.001$. Solid lines: current profiles for $\mathrm{La}_{t}=2, \mathrm{La}_{t}=1$, and $\mathrm{La}_{t}=0.5$ (from top to bottom). Dashed lines: extension of the asymptotes [with slope $\left(u_{*_{s}} / u_{*}\right) / \kappa$ ] corresponding to the currents at small depths to large depths. Dotted lines: extension of the asymptotes (with slope $1 / \kappa$ ) corresponding to the currents at large depths up to the depths where the currents would be zero, corresponding to the values of the apparent roughness length $k_{w} z_{0 w}$.

shows dramatically how $z_{0 w}$ may become various orders of magnitude larger than $z_{0}$ as $\mathrm{La}_{t}$ decreases (see further discussion below).

Note that, according to the present model, if measurements are taken at a range of depths well below the transition region located around $|z| \approx 1 /\left(\varepsilon k_{w}\right)$, the friction velocity corresponding to the total momentum flux $u *$ will be diagnosed correctly from the current profile, but the roughness length $z_{0}$ will be strongly overestimated as $z_{0 w}$. Conversely, if measurements are taken at a range of depths above this transition region (if that is feasible), $z_{0}$ will be correctly diagnosed from the current profile, but $u *$ will be underestimated as $u_{*}$. Data taken from an intermediate depth range coinciding with the transition between the two asymptotic portions of the profile (if they form a reasonably straight line on a logarithmic scale) will lead both to an overestimation of $z_{0}$ and to an underestimation of $u_{*}$. It is likely that at least one of these three possibilities occurs in a large fraction of the available field or laboratory measurements of wave-affected mean currents.

Circumstantial evidence that this is so is provided by the reported need to change (more specifically decrease) the value of the von Karmán constant to achieve an adequate collapse of measured current profiles in wall coordinates (Howe et al. 1982; Cheung and Street 1988; Craig and Banner 1994; Siddiqui and Loewen 2007), unless the friction velocity used to define $U^{+}$is that diagnosed from the current profile itself, here defined as $u_{*_{s}}$ (Siddiqui and Loewen 2007), which masks this problem. Clearly, neither of these procedures is very satisfactory, given their arbitrariness. More evidence supporting the discussion in the preceding paragraph is provided by the consistently high reported values of the roughness length diagnosed from current profiles, exceeding by orders of magnitude the value that would be expected from the morphology of the air-water interface, or the flow regime (Csanady 1984; Burchard 2001; Soloviev and Lukas 2003; Sullivan et al. 2004; Kudryavtsev et al. 2008). Yet more indications, of a more doubtful but suggestive nature, are provided by the fact that the slope of wave-affected currents plotted in wall-layer coordinates increases in some cases at larger depths [see, e.g., the diamond and circle symbols in Fig. 1 of Cheung and Street (1988), or the black circles and diamonds in Fig. 6 of Siddiqui and Loewen (2007)].

Although both a decrease of the friction velocity and an increase of the roughness length, as diagnosed from current profiles, might be expected as a result of vertical mixing of momentum due to wave breaking, the remarkable property of the model proposed here is that this phenomenon arises simply due to the partition of the shear stress imposed by nonbreaking waves, something that can be traced back to the production terms of the shear stress budget in (5), and is thus much easier to pinpoint physically. It is, of course, possible, and even likely, that both processes act in concert when wave breaking does occur, but it is striking that the present mechanism does not require wave breaking.

Figure 2 shows the variation of $u_{*_{s}} / u_{*}$ as a function of $\mathrm{La}_{t}$ for different values of the calibrating constant $\gamma$, from (9). Unsurprisingly, this ratio takes values that range from $\approx 1$ for large $\mathrm{La}_{t}$ to $\ll 1$ for small $\mathrm{La}_{t}$. Clearly, what matters for a correct representation of the variation in between is the value of $\gamma$, with large values corresponding to strong wave effects and small values to weaker wave effects. This partition of the friction velocity, or between the corresponding shear-induced and wave-induced stresses, is not an often measured or calculated quantity, but Fig. 5 of Bourassa (2000) presents an example with some relevance, even if a quantitative comparison is not easy. If an increase in wind speed is equated with a decrease of $\mathrm{La}_{t}$ (an idea that is suggested by the comparisons of the next subsection), and the ratio of the aqueous shear stress to the total atmospheric stress is equated with $u_{*} / u_{*}$ (which must at least be partially correct because the aqueous stress is estimated from current profiles), the decreasing trend of this ratio with increasing wind speed in Fig. 5 of Bourassa (2000) is consistent with Fig. 2. Another aspect that suggests this reasoning is sound is the leveling off of the stress ratio for the highest wind speeds in Fig. 5 of Bourassa (2000). 


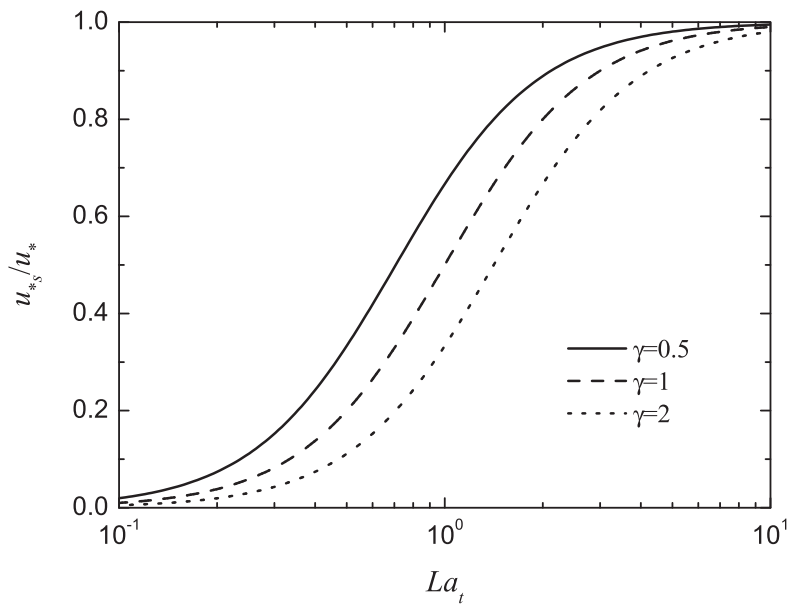

FIG. 2. Ratio of the shear-associated friction velocity to the total friction velocity as a function of $\mathrm{La}_{t}$ for different values of $\gamma$, calculated from (9). See legend for the meaning of different line types.

This is clearly consistent with a smaller sensitivity of $\mathrm{La}_{t}$ to the wind speed at the highest wind speeds, which is corroborated by the comparisons presented in the next subsection. Both results are compatible with the established idea that in well-developed seas in the real ocean, $\mathrm{La}_{t}$ becomes largely independent of the wind speed.

Figure 3 presents the variation of $k_{w} z_{0 w}$ and $z_{0 w} / z_{0}$ as a function of $\mathrm{La}_{t}$ from (21) for $\gamma=1$ and $\varepsilon=1$ (as assumed in Fig. 1) and different values of $k_{w} z_{0}$. As expected, $k_{w} z_{0 w}$ approaches $k_{w} z_{0}$ for large values of $\mathrm{La}_{t}$ but tends to a value independent of $k_{w} z_{0}$ at small $\mathrm{La}_{t}$. What this means is that at low $\mathrm{La}_{t}, z_{0 w}$ scales with $k_{w}^{-1}$ rather than with $z_{0}$, that is, $z_{0 w}$ is proportional to the wavelength of the dominant waves, not to any property of small-scale capillary waves (neglected in the model), or to the amplitude of the dominant waves $a_{w}$. This behavior is confirmed by the ratio $z_{0 w} / z_{0}$, which only approaches 1 for large values of $\mathrm{La}_{t}$, whereas it tends to be very high for small $\mathrm{La}_{t}$. As is consistent with the behavior of $k_{w} z_{0 w}, z_{0 w} / z_{0}$ at low $\mathrm{La}_{t}$ is inversely proportional to $k_{w} z_{0}$. Since in real situations $k_{w}$ $z_{0}$ may easily be as small as $10^{-5}$, the amplification of the apparent roughness length can be very pronounced. A qualitative comparison with Fig. 3 of Bourassa (2000) is pertinent. Although the dependence of $z_{0}$ (which should probably be taken as $z_{0 w}$ in the present notation) with $u_{*}$ in that figure cannot be tested quantitatively because wave information is missing, and the dependence on $u *$ affects both the true value of $z_{0}$ [see (26) below] and (21) via the definition of $\mathrm{La}_{t}$, the important point to retain from Fig. 3 of Bourassa (2000) is the enormous amplification of $z_{0}$. Bourassa (2000) notes that $z_{0}$ is about $10^{5}$ larger than expected from Charnock's relation (and therefore much higher than the values estimated for the true $z_{0}$ in the next subsection).
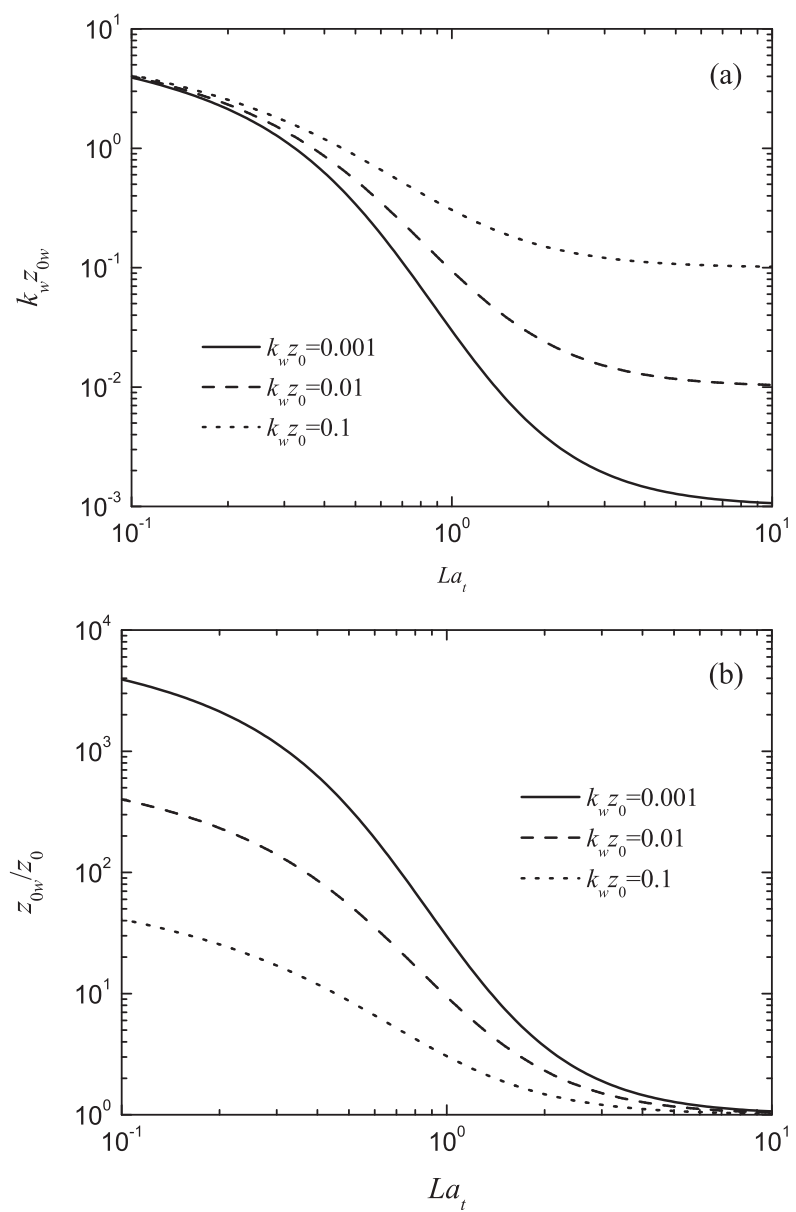

FIG. 3. Normalized apparent roughness length as a function of $\mathrm{La}_{t}$ for $\gamma=1$ and $\varepsilon=1$ from (21) for different values of $k_{w} z_{0}$. (a) Apparent roughness length normalized by $k_{w}$ and (b) ratio of apparent to true roughness length. See legend for the meaning of different line types.

\section{b. Comparison with Cheung and Street (1988)}

Finding adequate datasets to test the present model is challenging, because usually the quantities required as input to the model are not measured. First of all, measuring current profiles in the field with the required accuracy is extremely difficult, hence the most relevant studies typically involve laboratory experiments. Even in those cases, almost invariably not all relevant wave quantities are measured (Bourassa 2000; Siddiqui and Loewen 2007; Longo et al. 2012), and often the shear stress is not measured directly, but rather estimated from the current profiles (Bourassa 2000; Siddiqui and Loewen 2007), which makes comparisons more difficult [the erratic behavior of the current speeds measured by Siddiqui and Loewen (2007) as a function of the wind speed is another reason to exclude their data]. A notable exception is the laboratory experiments of Cheung and 
TABLE 1. Parameters of the datasets from Cheung and Street (1988) used here and derived parameters: wind speed, depth of the boundary layer $\delta$, wavelength of the dominant waves $\lambda_{w}$, depth of penetration of the wave stress $1 /\left(\varepsilon k_{w}\right)$, surface Stokes drift velocity $U_{S}(z=0)$, and turbulent Langmuir number $\mathrm{La}_{t}$. Parameters $1 /\left(\varepsilon k_{w}\right), U_{S}(z=0)$, and $\mathrm{La}_{t}$ were estimated from the dominant wave parameters using a monochromatic wave approximation (see text). Parameter $1 /\left(\varepsilon k_{w}\right)$ is estimated for the cases displayed in Figs. $4 \mathrm{~b}$ and 5a, where $\varepsilon=0.5$ (the lowest value of $\varepsilon$ considered). For other cases, $\varepsilon$ must be changed accordingly.

\begin{tabular}{ccccc}
\hline \hline Wind speed $\left(\mathrm{m} \mathrm{s}^{-1}\right)$ & $\delta(\mathrm{cm})$ & $\lambda_{w}(\mathrm{~cm})$ & $1 /\left(\varepsilon k_{w}\right)(\varepsilon=0.5)(\mathrm{cm})$ & $U_{S}(z=0)\left(\mathrm{cm} \mathrm{s}^{-1}\right)$ \\
\hline 2.6 & 31.0 & 4.2 & 1.3 & 0.015 \\
3.2 & 34.8 & 5.8 & 1.8 & 0.98 \\
4.7 & 26.4 & 12.7 & 4.1 & 2.6 \\
6.7 & 24.9 & 21.4 & 6.8 & 4.1 \\
9.9 & 35.4 & 27.1 & 8.6 & 6.0 \\
13.1 & 29.8 & 39.0 & 12.4 & 0.71 \\
\end{tabular}

Street (1988) of the current beneath surface waves generated by the wind. The relevant quantities are presented in their Table 1. As Kudryavtsev et al. (2008) do for the comparison presented in their Fig. 10, only wind-generated waves are considered here and the case among these waves with the lowest wind speed (where the wave amplitude is so small as to be barely measurable) is ignored.

The experiments with mechanical waves are excluded from this comparison because the assumption of the model that $d U / d z$ and $d U_{S} / d z$ have the same sign may not be strictly satisfied. The possibility that $d U / d z$ and $d U_{S} / d z$ have opposite signs has been demonstrated by Pearson (2018), for situations with weak (or no) wind, when turbulence exists beneath a wave field. This leads to a suppression of the instability to Langmuir circulations [which requires $(d U / d z)\left(d U_{S} / d z\right)>0$ ], modifying the stress partition assumed in (8), which relies on the existence of that instability (Teixeira 2011a).

For a reasonable range of input parameters, the present model predicted almost no difference between the current profiles beneath wind waves for the two lowest wind speeds in Table 1 of Cheung and Street (1988). This justifies (following Kudryavtsev et al. 2008) ignoring the profile for the lowest wind speed, $1.5 \mathrm{~m} \mathrm{~s}^{-1}$, which has a roughness length smaller than that expected for an aerodynamically smooth flow, and might be affected by some inaccuracy.

\section{1) UNBOUNDED MODEL}

The first comparison to be made uses an uncalibrated version of the "unbounded" model described in section 2c. The values of $u *$ from Table 1 of Cheung and Street (1988) are used directly in the model, the wave orbital velocity $a_{w} k_{w} c_{w}$ is taken as $\sqrt{2}\left(\tilde{\tilde{u}}_{0}^{2}\right)^{1 / 2}$, where $\left(\tilde{\tilde{u}}_{0}^{2}\right)^{1 / 2}$ is the root-mean-square orbital velocity in the data [as is consistent with (4) and (5) of Cheung and Street (1988), where $\hat{\eta}_{S}$ is equivalent to $a_{w}$ here], the angular frequency $\sigma_{w}$ is equated to $2 \pi f_{D}$, where $f_{D}$ is the frequency (in cycles) of the dominant waves, and the corresponding wavenumber is $k_{w}=\sigma_{w}^{2} / g$ from the linear dispersion relation of deep-water surface gravity waves. Some key parameters are presented in Table 1 . An evidently crucial detail is how to define $z_{0}$. As a first approximation the definition valid for aerodynamically smooth flow is adopted: $z_{0}=0.11 \nu / u *$ (Kraus and Businger 1994), with $\nu=10^{-6} \mathrm{~m}^{2} \mathrm{~s}^{-1}$. Figure 4 shows a comparison of the model with the data presented in Fig. 1 of Cheung and Street (1988) (excluding the upward-pointing triangles for the reasons explained above), assuming $\varepsilon=2$ and $\gamma=2$, as in Teixeira (2012) (Fig. 4a) and using the adjusted values $\varepsilon=0.5$ and $\gamma=0.5$ (Fig. $4 \mathrm{~b}$ ).

It can be seen in Fig. 4a that the behavior of the measured currents is reasonably well reproduced qualitatively, with a decrease of the overall normalized current speed as the wind speed increases. In terms of the input parameters of the model, this is due to a decrease of the turbulent Langmuir number $\mathrm{La}_{t}$ as the wind speed increases for the lowest wind speeds, but mostly due to an increase in penetration of the wave motion at the highest wind speeds, for which $\mathrm{La}_{t}$ actually changes very little (see Table 1). Noteworthy disagreements are that the range of variation of the current speed in the model is much too wide compared with the data, in particular, the current speed in wall coordinates is overestimated for the lowest wind speed and quite underestimated for the highest wind speeds. Additionally, although two logarithmic portions of the current profile exist in the model at the highest wind speeds (lowest values of $\mathrm{La}_{t}$ ), these portions do not coincide with the data that show a reduced slope (e.g., stars and open circles). Finally, the detailed variation with the wind speed is not reproduced. While most of the variation occurs at the lowest wind speeds in the model and weakens roughly monotonically as the wind speed increases, the rate of variation seems to increase again at the highest wind speeds in the data.

When Fig. 4a is compared with Fig. 10 of Kudryavtsev et al. (2008), it may be noticed that the agreement with the data is somewhat less satisfactory. Although the performance of the model of Kudryavtsev et al. (2008) is 

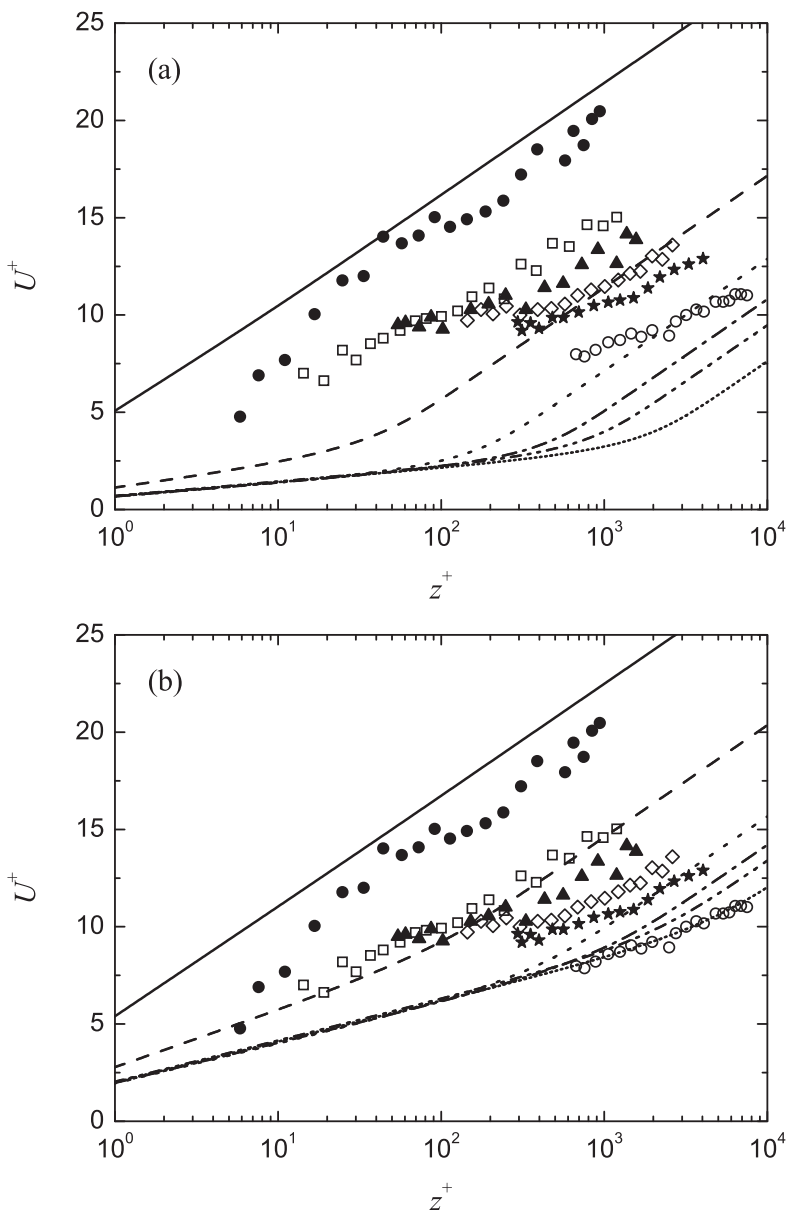

FIG. 4. Comparison between normalized current speed profiles in wall coordinates from the model developed here, given by (16) or (17) (lines), and from the measurements of Cheung and Street (1988) (symbols), for different wind speeds. The model assumes $c_{1}=0.11$ and $c_{2}=0$ in (26). Solid line and filled circles, $2.6 \mathrm{~m} \mathrm{~s}^{-1}$; dashed line and squares, $3.2 \mathrm{~m} \mathrm{~s}^{-1}$; dotted line and triangles, $4.7 \mathrm{~m} \mathrm{~s}^{-1}$; dashdotted line and diamonds, $6.7 \mathrm{~m} \mathrm{~s}^{-1}$; dash-double-dotted line and stars, $9.9 \mathrm{~m} \mathrm{~s}^{-1}$; short-dotted line and open circles, $13.1 \mathrm{~m} \mathrm{~s}^{-1}$. The model assumes (a) $\gamma=2$ and $\varepsilon=2$, and (b) $\gamma=0.5$ and $\varepsilon=0.5$.

itself far from perfect, its consideration of the effect of the viscous boundary layer for the current profile with the lowest wind speed substantially improves the agreement at small depths compared with the present model. Additionally, the model of Kudryavtsev et al. (2008) does not underestimate the current as much at the highest wind speeds. Curiously, it has some deficiencies similar to those of the present model, namely, it overestimates the sensitivity of the normalized current to the wind speed at intermediate values of that parameter and, on the contrary, has a too weak dependence for the highest values. On the other hand, the model of Kudryavtsev et al. (2008) is unable to capture the apparent reduction of $u *$ by the wave stress, but a somewhat similar effect is mimicked by the transition of the profiles to their viscous boundary layer form (also partly affected by wave breaking).

Clearly, the comparison presented in Fig. 4a indicates an overestimation of parameter $\gamma$ in the present model. One might wonder why this happens, given that this calibration seemed to work for predictions of the dissipation rate by Teixeira (2012), and also in his preliminary calibration procedure using current profiles from the LES of Li et al. (2005). Possible reasons are speculative, but might have to do with inadvertently accounting for the effect of wave breaking in the first case and adopting a value of $\gamma$ suitable for monochromatic waves in the second, both conditions which are not applicable here. It seems fortuitous that both of these distinct differences should lead to a similar value of $\gamma$.

To improve agreement with the data of Cheung and Street (1988), $\gamma$ and $\varepsilon$ may be readjusted. Figure $4 \mathrm{~b}$ shows a comparison similar to that of Fig. 4a, but where $\gamma=0.5$ and $\varepsilon=0.5$ are assumed, presumably to account for both the absence of wave breaking in the experiments of Cheung and Street (1988) and the fact that the waves are nonmonochromatic. The adjusted values of these parameters improve the agreement, particularly for the dataset with the highest wind speed (making it almost perfect by construction), but this turns out not to be sufficient. The variation of the normalized current speed for intermediate wind speeds is still affected by the problems pointed out above.

It is likely that the flow in the experiments under consideration was not always aerodynamically smooth, but rather became aerodynamically rough at the highest wind speeds, because of the small-scale corrugations forced at the air-water interface by the wind stress. A form of the roughness length that reflects this is

$$
z_{0}=c_{1} \frac{\nu}{u_{*}}+c_{2} \frac{u_{*}^{2}}{g}
$$

where $c_{1}$ and $c_{2}$ are coefficients, and the second term is of a form analogous to the Charnock relation but using the friction velocity in the water. In what follows, $\gamma, \varepsilon, c_{1}$, and $c_{2}$ are adjusted to produce the best possible agreement with the data of Cheung and Street (1988). The values found for the unbounded model are $\gamma=0.25$, $\varepsilon=0.5, c_{1}=0.2$, and $c_{2}=0.9$.

Figure 5a shows a comparison of the model with the data of Cheung and Street (1988) using these adjusted parameters. The agreement is much better than in Fig. 4, in particular for the rate of variation of the normalized current profiles at intermediate wind speeds (this is not surprising, being a result of the calibration procedure). Agreement is less close for the lowest wind speed considered at small depths, due to the absence of a viscous 

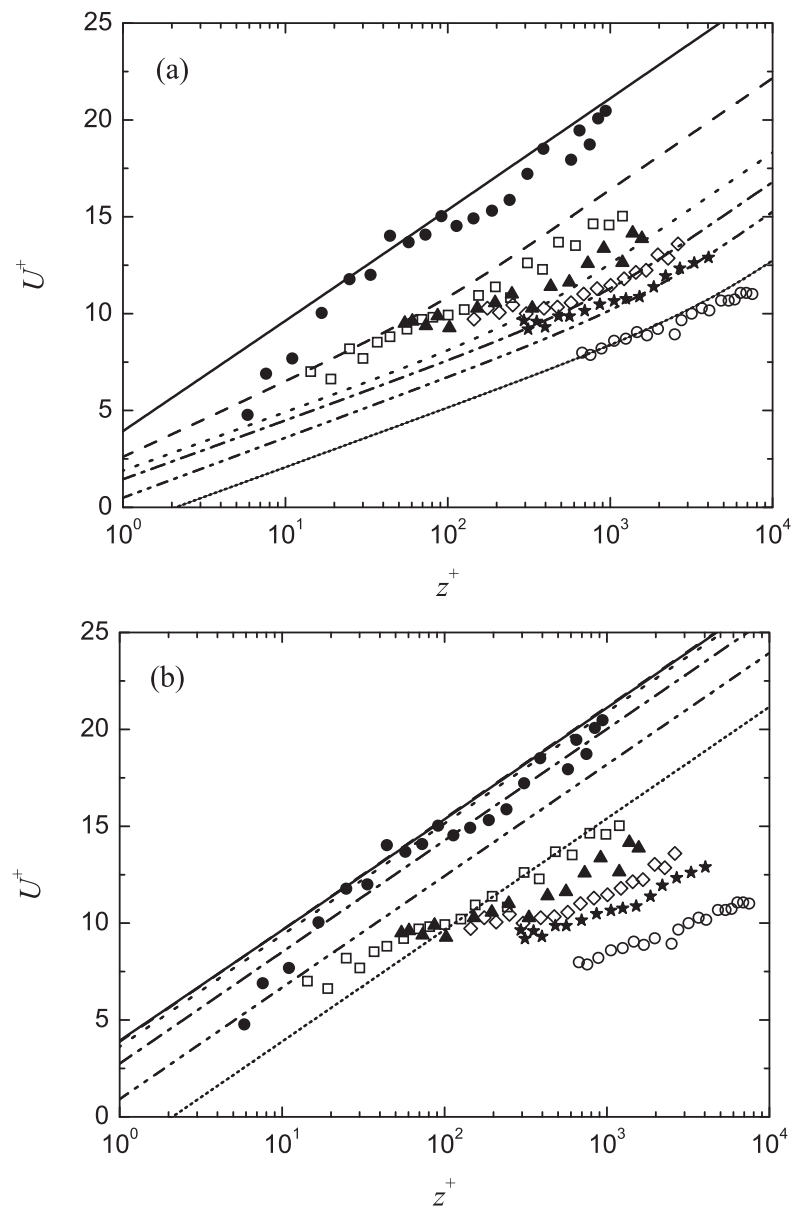

FIG. 5. As in Fig. 4, but for $c_{1}=0.2$ and $c_{2}=0.9$ in (26), and different values of $\gamma$ and $\varepsilon$. The model assumes (a) $\gamma=0.25$ and $\varepsilon=0.5$, and (b) $\gamma=0$ (i.e., no wave effects).

boundary layer in the model, but this is a minor limitation. The transition of the datasets from a slope corresponding to $u *$ to the smaller value corresponding to $u *$ is fairly well reproduced, occurring somewhere around between the wind speeds of 4.7 and $6.7 \mathrm{~m} \mathrm{~s}^{-1}$. However, at these intermediate wind speeds, the current at the smallest depths covered by the data is somewhat underestimated by the model (the shear suggested by the data at those depths is weaker than expected). Additionally while the current is slightly underestimated for a wind speed of $4.7 \mathrm{~m} \mathrm{~s}^{-1}$, it is on the contrary slightly overestimated for a wind speed of $3.2 \mathrm{~m} \mathrm{~s}^{-1}$. It is perhaps risky to attach too much relevance to these discrepancies in detail, given the limited precision of the measurements (which are, nevertheless, among the most precise that could be found).

The value of $\gamma$ was already discussed above. The value of $\varepsilon$ adopted for this comparison would correspond to the Stokes drift of a monochromatic wave with a wavelength 4 times larger than the wavelength of the dominant waves, obtained from the data. The significance of this mismatch for nonmonochromatic waves (such as the ones under consideration) is not obvious, but indicates a larger depth of penetration of the waveinduced stress than would be expected. The Stokes drift gradient of a wave spectrum is known to be characterized by a larger penetration depth than a monochromatic wave with the same dominant wavelength (Fig. 18 of Li and Garrett 1993), and this may perhaps account for a similar effect on the wave-induced stress.

Concerning parameters estimated for $(26), c_{1}=0.2$ is substantially larger than the value of 0.11 most commonly accepted for aerodynamically smooth flow. It is worth noting that, in Fig. 10 of Kudryavtsev et al. (2008), the thin line (corresponding to aerodynamically smooth flow) assumes $z_{0}=0.18 v / u *$, which is not too different from the value employed here. Regarding $c_{2}$, the Charnock relation, when expressed in terms of the friction velocity in the airflow, usually has a coefficient of 0.015 . Taking into account continuity of the shear stress at the air-water interface, when that relation is expressed in terms of the friction velocity in the water, the coefficient should become $833 \times 0.015=12.5$. This is clearly much larger than $c_{2}=0.9$ used here, but it should be noted that the Charnock relation, as usually formulated, is valid in the open ocean and for a fully developed wave field, which are very distinct conditions from those produced in the experiments of Cheung and Street (1988). Additionally, continuity of the shear stress at the air-water interface (used in the above calculation) assumes equilibrium, which is not warranted in these experiments either. Nevertheless, a reassuring aspect is that, on dimensional grounds, the quantities on which (26) depends are still likely to be the most relevant.

It might be argued that the agreement between model and measurements in Fig. 5a was artificially improved by allowing $z_{0}$ to vary according to (26). To test this, Fig. 5b shows a similar comparison, but where wave effects are ignored altogether, and only the dependence of $z_{0}$ on $u_{*}$ via (26) is retained (with similar values of $c_{1}$ and $c_{2}$ ). It is clear that this dependence, by itself, is unable to produce a satisfactory agreement with the measurements, particularly at the highest wind speeds, and naturally does not represent the decrease of the apparent value of $u *$, although it does represent a part of the increase of $z_{0}$ required to match the data. Relatedly, (26) contributes significantly to the weakening of the current speed at the highest wind speeds, which is important to improve agreement with the data relative to Fig. 4.

\section{2) FINITE-DEPTH MODEL}

Figure 6 shows a similar comparison to Fig. 5, but using the finite-depth model developed at the end of 


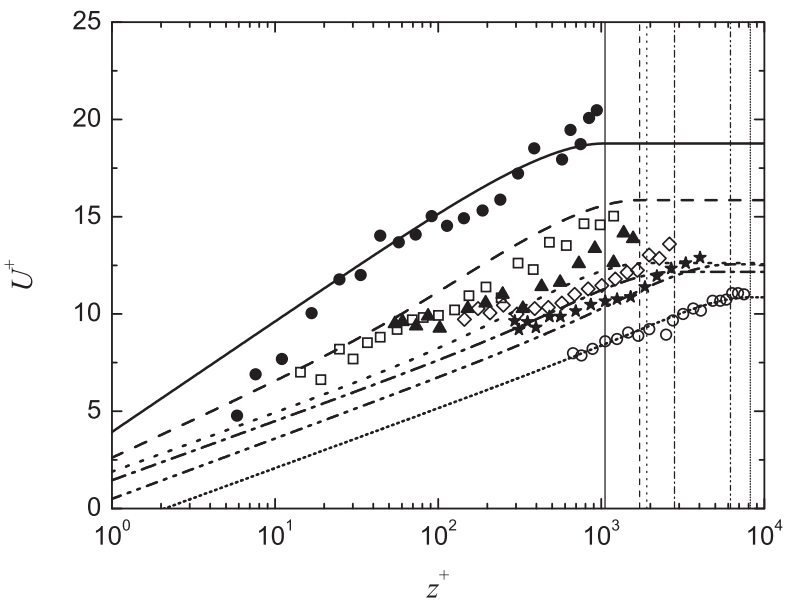

FIG. 6. As in Fig. 5, but using the finite-depth model for which the shear stress decreases linearly with depth (24), with $\gamma=0.25, \varepsilon=1$, $c_{1}=0.2$, and $c_{2}=0.9$. The vertical lines (same type as the corresponding current profiles) denote the depths at which the shear stress reaches zero in each case $\left(z^{+}=\delta u * / \nu\right)$. Note that the current profiles for larger depths remain constant in the model.

section 2c. Because of the log-linear form of the current profile, the current solutions are no longer composed of straight line segments when using a logarithmic depth scale but tend to have a reduction in shear at the depths near where the shear stress becomes zero (and the current speed stabilizes), marked by the vertical lines in Fig. 6 . Below those levels the shear obviously becomes zero, as is denoted by the horizontal lines in Fig. 6. However, some modified form of the current slope transition at depth $|z| \approx 1 /\left(\varepsilon k_{w}\right)$ still holds, as can be inferred from Fig. 6 , if that depth is above the level where the shear stress vanishes [which always happens in the data of Cheung and Street (1988); see Table 1 herein]. The parameter values used in Fig. 6 are $\gamma=0.25, \varepsilon=1, c_{1}=0.2$, and $c_{2}=0.9$. The agreement between the model and measurements is roughly as satisfactory as in Fig. 5, with essentially the same deficiencies in the midrange of wind speeds. At the largest depths considered (near to $|z|=\delta$ ) the model tends to underestimate the measurements more, perhaps because the reduction of shear in those regions is too large due to the assumption of a linearly decreasing shear stress. In reality, the fact that the shear stress decays to zero more gradually might explain why no marked reduction in the shear is detectable in the data at those depths. The existence of this shear reduction in the model counteracts the transition to a larger shear that occurs below the depth $|z| \approx 1 /\left(\varepsilon k_{w}\right)$, when this is not too distant from $|z|=\delta$. This is what allows a larger value of $\varepsilon$ to be employed in Fig. 6.

A noteworthy property of this finite-depth model is that it enables an estimation of the magnitude of the surface current speed $U_{0}$, as noted at the end of section $2 \mathrm{c}$.

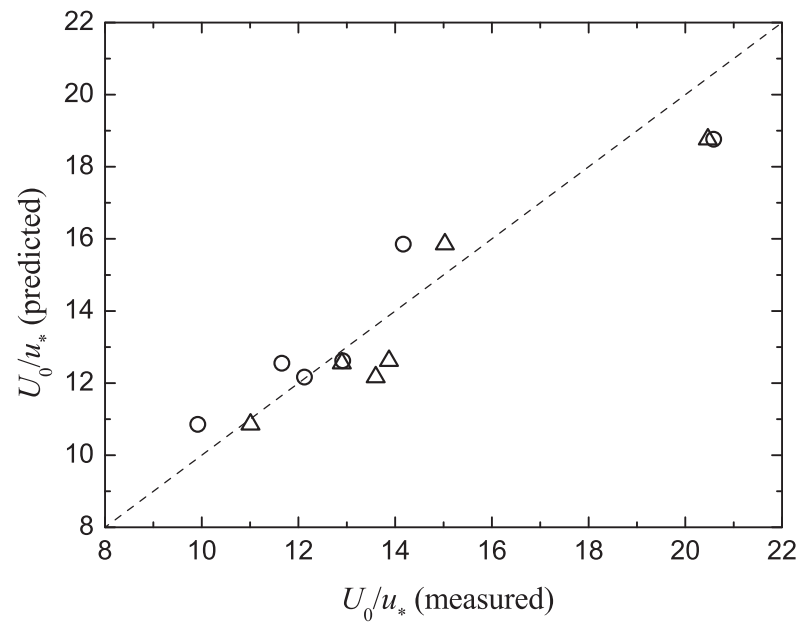

FIG. 7. Normalized current speed at the surface predicted by the finite-depth model in (24) (for $z^{+}=\delta u_{*} / \nu$ ) as a function of corresponding values derived from the measurements. Circles are measured values taken directly from Table 1 of Cheung and Street (1988); triangles are measured values taken as the data point at the largest depth from the datasets corresponding to each different wind speed.

Figure 7 shows a comparison of the values of $U_{0} / u *$ calculated from the model (corresponding to the horizontal portions of the curves in Fig. 6) with the values that can be either obtained directly from Table 1 of Cheung and Street (1988) (circles), or obtained from the data point with the largest depth in the datasets for each wind speed in Fig. 6 (triangles). It can be seen that the agreement is encouraging, with correlation coefficients of $\approx 0.95$ in both cases, although the model does tend to systematically underestimate the data. However, given the strong assumptions adopted, the agreement is surprisingly good.

\section{Concluding remarks}

This study presents a simple model for the wind-driven current existing in the oceanic boundary layer in the presence of surface waves generated by the wind. The model sheds light on two puzzling aspects that have been noted repeatedly about these currents, for which a logarithmic profile model, with the friction velocity $u_{*}$ and roughness length $z_{0}$ as basic parameters, has often been adopted. First, if the current speed is scaled using the total friction velocity, measured independently, for example, using the surface wind stress, the friction velocity diagnosed from shear in the current profile is smaller than expected, being only a fraction of the total friction velocity. Second, the roughness length diagnosed from the same fitting procedure is much larger than expected, by various orders of magnitude, being inconsistent with the roughness length that would be estimated either for 
an aerodynamically smooth flow, or aerodynamically rough flow affected by waves. The corresponding Charnock parameter appears to be enormously amplified (Bourassa 2000).

Both of these features are explained here as resulting from a partition of the total turbulent shear stress into a shear-induced component and a waveinduced component, which result from the local mechanical production of this stress by the mean shear in the current profile, and by the Lagrangian strain rate associated Stokes drift of the waves, respectively, when the effect of nonbreaking waves is included in the equations of motion via the Craik-Leibovich vortex force. In this framework, the wave-associated part of the shear stress is not a property of the wave itself, as assumed by some authors, but is a stress created on the turbulence (which coexists with the shear-induced stress) by Stokes drift straining of turbulent vorticity into the streamwise direction (the assumed direction of both the mean current and the Stokes drift; Teixeira 2011a). This is independent from any vertical mixing associated with preexisting turbulence, or turbulence injected into the water by wave breaking.

It is likely that this mechanism associated with nonbreaking waves acts in concert with other mechanisms related to wave breaking, and with the transport of turbulence by itself in general, but the fact that it can account for the two phenomena mentioned above, and that its dependence on the turbulent Langmuir number appears to be confirmed by measurements, support its relevance.

The model predicts that the part of the turbulent shear stress induced by shear in the surface layer becomes a progressively smaller fraction of the total stress near the surface and down to a depth of the order the wavelength of the dominant surface waves as $\mathrm{La}_{t}$ decreases. This leads to the perceived reduction of the friction velocity. The model also predicts that the roughness length inferred if the uppermost portion of the current profile is disregarded is amplified by various orders of magnitude as $\mathrm{La}_{t}$ decreases, and scales with $k_{w}^{-1}$, that is, the wavelength of the waves, at small $\mathrm{La}_{t}$. The profile of the windinduced current becomes flatter (i.e., less different from its surface value) as $\mathrm{La}_{t}$ decreases.

If the parameters in the model are adjusted appropriately, departing from their values assumed in Teixeira (2012) (presumably to account for the facts that there is no substantial wave breaking in the experiments and the waves are not monochromatic), good agreement is found with the laboratory measurements of Cheung and Street (1988), which appear to be the only dataset that is precise and comprehensive enough for this purpose. Other more recent datasets (Siddiqui and Loewen 2007; Longo et al. 2012) either seem unreliable, or do not provide complete enough information about the characteristics of the wave field or of the total shear stress. In the experiments of Cheung and Street (1988), the current profile becomes flatter as the wind speed increases. Using the present model, this is interpreted as being primarily due to a decrease in $\mathrm{La}_{t}$ at the lowest wind speeds, and due to an increasingly deeper penetration of the wave stress, conjugated with a higher real roughness length, at the highest wind speeds.

As in the present model, a recent study of Sinha et al. (2015) uses insights from Teixeira (2012) to develop a turbulence closure that includes wave effects. However, the dataset they use to test their model, from the LES of Tejada-Martinez et al. (2013), refers to shallow water flow, and is thus strongly affected by the bottom boundary layer. Sinha et al. (2015) primarily focus on an analysis of the current profile in wall coordinates within the bottom boundary layer, but the full-depth current profiles shown by them (e.g., their Figs. 19 and 21) suggest a relatively modest agreement between their model in the top boundary layer adjacent to the airwater interface, despite the fact that they include a term in the shear stress definition that is nonlocal, accounting for turbulent transport of TKE (which is not considered here).

To bring the model presented here closer to real oceanic conditions, and thus increase its usefulness, it is probably not only necessary to account for nonlocal mixing (which is important in some datasets), but also for the effect of Earth's rotation, as wind-driven currents are known to be typically misaligned with the surface stress and rotate with depth, in accordance with Ekman layer theory. However, within the surface layer where the shear stress is the primary mechanism shaping the current, shear at least is necessarily aligned with the wind stress, and thus the model presented here may still be directly applicable to the streamwise component of the current.

Defining precisely the range of applicability of the present model is complicated (when compared to the atmosphere) by the presence of surface waves, as their influence may in some cases be confined to the oceanic surface layer (as happens here), and in others extend below it. To a first approximation, the surface layer might be defined as the layer in which there is little fractional change in the vertical of both the shear stress and the current direction.

The results reported here are presented in dimensionless form, which should facilitate their transposition to real oceanic conditions, enabling the development of physically based parameterizations for the turbulent 
momentum flux in the wave-affected boundary layer for ocean circulation models.

\section{REFERENCES}

Agrawal, Y. C., E. A. Terray, M. A. Donelan, P. A. Hwang, A. J. Williams III, W. M. Drennan, K. K. Kahma, and S. A. Kitaigorodskii, 1992: Enhanced dissipation of kinetic energy beneath surface waves. Nature, 359, 219-220, https://doi.org/ 10.1038/359219a0.

Bourassa, M. A., 2000: Shear stress model for the aqueous boundary layer near the air-water interface. J. Geophys. Res., 105, 1167-1176, https://doi.org/10.1029/1999JC900250.

Burchard, H., 2001: Simulating the wave-enhanced layer under breaking surface waves with two-equation turbulence models. J. Phys. Oceanogr., 31, 3133-3145, https://doi.org/10.1175/ 1520-0485(2001)031<3133:STWELU>2.0.CO;2.

Cheung, T. K., and R. L. Street, 1988: The turbulent layer in the water at an air-water interface. J. Fluid Mech., 194, 133-151, https://doi.org/10.1017/S0022112088002927.

Craig, P. D., 1996: Velocity profiles and surface roughness under breaking waves. J. Geophys. Res., 101, 1265-1277, https:// doi.org/10.1029/95JC03220.

_ and M. L. Banner, 1994: Modeling wave-enhanced turbulence in the ocean surface layer. J. Phys. Oceanogr., 24, 2546-2559, https:// doi.org/10.1175/1520-0485(1994)024<2546:MWETIT>2.0.CO;2.

Csanady, G. T., 1984: The free surface turbulent shear layer. J. Phys. Oceanogr., 14, 402-411, https://doi.org/10.1175/ 1520-0485(1984)014<0402:TFSTSL $>2.0$. CO;2.

_ 2004: Air-Sea Interaction: Laws and Mechanisms. Cambridge University Press, 239 pp.

Drennan, W. M., M. A. Donelan, E. A. Terray, and K. B. Katsaros, 1996: Oceanic turbulence dissipation measurements in SWADE. J. Phys. Oceanogr., 26, 808-815, https://doi.org/ 10.1175/1520-0485(1996)026<0808:OTDMIS > 2.0.CO;2.

Feddersen, F., J. H. Trowbridge, and A. J. Williams III, 2007: Vertical structure of dissipation in the nearshore. J. Phys. Oceanogr., 37, 1764-1777, https://doi.org/10.1175/JPO3098.1.

Gargett, A. E., 1989: Ocean turbulence. Annu. Rev. Fluid Mech., 21, 419-451, https://doi.org/10.1146/annurev.fl.21.010189.002223.

Gemmrich, J. R., and D. M. Farmer, 1999: Near-surface turbulence and thermal structure in a wind-driven sea. J. Phys. Oceanogr., 29, 480-499, https://doi.org/10.1175/1520-0485(1999) $029<0480$ :NSTATS $>2.0 . \mathrm{CO} ; 2$.

Gerbi, G. P., J. H. Trowbridge, E. A. Terray, A. J. Pluddeman, and T. Kukulka, 2009: Observations of turbulence in the ocean surface boundary layer: Energetics and transport. J. Phys. Oceanogr., 39, 1077-1096, https://doi.org/10.1175/2008JPO4044.1.

Grant, A. L. M., and S. E. Belcher, 2009: Characteristics of Langmuir turbulence in the ocean mixed layer. J. Phys. Oceanogr. 39, 1871-1887, https://doi.org/10.1175/2009JPO4119.1.

Harcourt, R. R., 2013: A second-moment closure model of Langmuir turbulence. J. Phys. Oceanogr., 43, 673-697, https:// doi.org/10.1175/JPO-D-12-0105.1.

Howe, B. M., A. J. Chambers, S. P. Klotz, T. K. Cheung, and R. L. Street, 1982: Comparison of profiles and fluxes of heat and momentum above and below an air-water interface. J. Heat Transfer, 104, 34-39, https://doi.org/10.1115/1.3245064.

Jones, N. L., and S. G. Monismith, 2008: The influence of whitecapping waves on the vertical structure of turbulence in a shallow estuarine embayment. J. Phys. Oceanogr., 38, 1563 1580, https://doi.org/10.1175/2007JPO3766.1.
Kondo, J., 1976: Parameterization of turbulent transport in the top meter of the ocean. J. Phys. Oceanogr., 6, 712-720, https:// doi.org/10.1175/1520-0485(1976)006<0712:POTTIT>2.0.CO;2.

Kraus, E. B., and J. A. Businger, 1994: Atmosphere-Ocean Interaction. 2nd ed. Oxford University Press, $362 \mathrm{pp}$.

Kudryavtsev, V., V. Shrira, V. Dulov, and V. Malinovsky, 2008: On the vertical structure of wind-driven sea currents. J. Phys. Oceanogr., 38, 2121-2144, https://doi.org/10.1175/2008JPO3883.1.

Kukulka, T., and R. R. Harcourt, 2017: Influence of Stokes drift decay scale on Langmuir turbulence. J. Phys. Oceanogr., 47, 1637-1656, https://doi.org/10.1175/JPO-D-16-0244.1.

— A. J. Plueddemann, J. H. Trowbridge, and P. P. Sullivan, 2010: Rapid mixed layer deepening by the combination of Langmuir and shear instabilities: A case study. J. Phys. Oceanogr., 40, 2381-2400, https://doi.org/10.1175/2010JPO4403.1.

Lee, M. J., J. Kim, and P. Moin, 1990: Structure of turbulence at high shear rate. J. Fluid Mech., 216, 561-583, https://doi.org/ 10.1017/S0022112090000532.

Li, M., and C. Garrett, 1993: Cell merging and the jet/downwelling ratio in Langmuir circulation. J. Mar. Res., 51, 737-769, https:// doi.org/10.1357/0022240933223945.

—,- , and E. Skyllingstad, 2005: A regime diagram for classifying turbulent large eddies in the upper ocean. Deep-Sea Res., 52, 259-278, https://doi.org/10.1016/j.dsr.2004.09.004.

Longo, S., D. Liang, L. Chiapponi, and L. A. Jiménez, 2012: Turbulent flow structure in experimental laboratory wind-generated gravity waves. Coast. Eng., 64, 1-15, https://doi.org/10.1016/ j.coastaleng.2012.02.006.

McLeish, W. L., and G. E. Putland, 1975: Measurements of winddriven flow profiles in the top millimeter of water. J. Phys. Oceanogr., 5, 516-518, https://doi.org/10.1175/1520-0485(1975) 005<0516:MOWDFP $>2.0 . \mathrm{CO} ; 2$.

McWilliams, J. C., P. P. Sullivan, and C.-H. Moeng, 1997: Langmuir turbulence in the ocean. J. Fluid Mech., 334, 1-30, https:// doi.org/10.1017/S0022112096004375.

Pearson, B., 2018: Turbulence-induced anti-Stokes flow and the resulting limitations of large-eddy simulation. J. Phys. Oceanogr., 48, 117-122, https://doi.org/10.1175/JPO-D-17-0208.1.

Phillips, O. M., 1977: The Dynamics of the Upper Ocean. Cambridge University Press, $336 \mathrm{pp}$.

Polton, J. A., and S. E. Belcher, 2007: Langmuir turbulence and deeply penetrating jets in an unstratified mixed layer. J. Geophys. Res., 112, C09020, https://doi.org/10.1029/ 2007JC004205.

- D. M. Lewis, and S. E. Belcher, 2005: The role of waveinduced Coriolis-Stokes forcing on the wind-driven mixed layer. J. Phys. Oceanogr., 35, 444-457, https://doi.org/10.1175/ JPO2701.1.

Rascle, N., and F. Ardhuin, 2009: Drift and mixing under the ocean surface revisited: Stratified conditions and model-data comparisons. J. Geophys. Res., 114, C02016, https://doi.org/10.1029/ 2007JC004466.

,-- , and E. A. Terray, 2006: Drift and mixing under the ocean surface: A coherent one-dimensional description with application to unstratified conditions. J. Geophys. Res., 111, C03016, https://doi.org/10.1029/2005JC003004.

Siddiqui, M. H. K., and M. R. Loewen, 2007: Characteristics of the wind drift layer and microscale breaking waves. J. Fluid Mech., 573, 417-456, https://doi.org/10.1017/S0022112006003892.

Sinha, N., A. E. Tejada-Martinez, C. Akan, and C. E. Grosch, 2015: Toward a K-profile parameterization of Langmuir turbulence in shallow coastal shelves. J. Phys. Oceanogr., 45, 2869-2895, https://doi.org/10.1175/JPO-D-14-0158.1. 
Soloviev, A., and R. Lukas, 2003: Observation of wave-enhanced turbulence in the near-surface layer of the ocean during TOGA COARE. Deep-Sea Res. I, 50, 371-395, https://doi.org/ 10.1016/S0967-0637(03)00004-9.

Sullivan, P. P., J. C. McWilliams, and W. K. Melville, 2004: The oceanic boundary layer driven by wave breaking with stochastic variability. Part 1. Direct numerical simulations. J. Fluid Mech., 507, 143-174, https://doi.org/10.1017/S0022112004008882.

Teixeira, M. A. C., 2011a: A linear model for the structure of turbulence beneath surface water waves. Ocean Modell., 36, 149162, https://doi.org/10.1016/j.ocemod.2010.10.007.

_ 2011b: On the connection between dissipation enhancement in the ocean surface layer and Langmuir circulations. J. Phys. Oceanogr., 41, 2000-2007, https://doi.org/10.1175/JPO-D-11-062.1.

__ 2012: The influence of Langmuir turbulence on the scaling for the dissipation rate in the oceanic boundary layer. J. Geophys. Res., 117, C05015, https://doi.org/10.1029/2011JC007235.

— progressive surface wave. J. Fluid Mech., 458, 229-267, https:// doi.org/10.1017/S0022112002007838.

_ , and - 2010: On the structure of Langmuir turbulence. Ocean Modell., 31, 105-119, https://doi.org/10.1016/j.ocemod.2009.10.007.
Tejada-Martinez, A. E., C. Akan, C. E. G. N. Sinha, and G. Martinat, 2013: Surface dynamics in les of full-depth Langmuir circulation in shallow water. Phys. Scr., 2013, 014008, https://doi.org/10.1088/0031-8949/2013/T155/ 014008.

Terray, E. A., M. A. Donelan, Y. C. Agrawal, W. M. Drennan, K. K. Kahma, A. J. Williams III, P. A. Hwang, and S. A. Kitaigorodskii, 1996: Estimates of kinetic energy dissipation under breaking waves. J. Phys. Oceanogr., 26, 792-807, https://doi.org/10.1175/ 1520-0485(1996)026<0792:EOKEDU>2.0.CO;2.

— W. M. Drennan, and M. A. Donelan, 1999: The vertical structure of shear and dissipation in the ocean surface layer. Proc. Symp. on the Wind-Driven Air-Sea Interface: Electromagnetic and Acoustic Sensing, Wave Dynamics and Turbulent Fluxes, Sydney, Australia, University of New South Wales, 239-245.

Thorpe, S. A., 2005: The Turbulent Ocean. Cambridge University Press, 439 pp.

Van Roekel, L. P., B. Fox-Kemper, P. P. Sullivan, P. E. Hamlington, and S. R. Haney, 2012: The form and orientation of Langmuir cells for misaligned winds and waves. J. Geophys. Res., 117, C05001, https://doi.org/10.1029/2011JC007516. 